\title{
Dynamics of Marangoni Convection in Hybrid Nanomaterials Flow With Dust Particles Random Motion
}

\author{
M. ljaz Khan ( $\nabla$ mikhan@math.qau.edu.pk ) \\ Riphah International University
}

\section{Research Article}

Keywords: Dust particles, hybrid nanofluid, Marangoni convection, MHD, Porous medium, numerical method

Posted Date: April 29th, 2021

DOI: https://doi.org/10.21203/rs.3.rs-456333/v1

License: (a) (i) This work is licensed under a Creative Commons Attribution 4.0 International License.

Read Full License 


\title{
Dynamics of Marangoni Convection in Hybrid Nanomaterials Flow with Dust Particles Random Motion
}

\author{
M. Ijaz Khan \\ Department of Mathematics and Statistics, Riphah International University I-14, Islamabad \\ 44000, Pakistan
}

\section{("Corresponding author email: mikhan@math.qau.edu.pk (M. I. Khan))}

\begin{abstract}
Here we are working on the flow of dust particles in hybrid nanofluid. Marangoni convective flow of hybrid nanofluid is accounted by considering silver and copper as nanoparticles and water as base fluid. Dust particles and nanoparticles are used in this flow are spherical type. For thermal conductivity we have considered the Maxwell model. Porous medium is placed over a stretching sheet. Flow is generated via stretching sheet. MHD effects are also considered. Nonlinear equation of fluid phase and dust phase are converted in to ODE's by suitable transformations. These ordinary differential equations are solved numerically. Effect of involved dimensionless variables against velocity and temperature of hybrid nanofluid and dust phase, skin friction and Nusselt number of hybrid nanofluid is studied through graphs and tables. It is observed that temperature and velocity is more in case of hybrid nanofluid as compared to dust phase. Velocity of $\mathrm{Ag}-\mathrm{Cu}$ water hybrid nanofluid enhances for greater mass concentration of dust particles. Velocity in both phase decay for higher porosity variable. Good match of results are seen by comparing current situation to earlier study in particular case.
\end{abstract}

Keywords: Dust particles; hybrid nanofluid; Marangoni convection; MHD; Porous medium; numerical method.

\section{1: Introduction}


Heat and momentum transfer finds wide range of applications in sciences and engineering. In the last few years, different researchers have worked on studying the heat and mass transfer in dusty and nanofluids. In this study the variation of mass and heat transfer of nanofluids on the stretching surfaces, volume fraction of nanofluids is taken in consideration which area embedded with dust particles. The purpose of this study is to find the metallic oxides and metals while they are embedded with various nanofluids to get good thermal conductivity. There are wide ranges of applications of convective flow of viscous dusty fluids in combustion, power plant piping, waste water treatment etc. Nanofluids have wide range of applications in heat transfer in various energy systems. Choi et. al was the first one who studied nanofluids. He studied the enhancement of thermal conductivity by embedding the base fluid and nanoparticles [1]. Marble et. al focuses his study on the effect of fluid mechanics and dusty gases and vice versa [2]. Chakrabarti and Gupta et. al analysis the Magneto Hydro Dynamics and characteristics of heat transfer on the stretching surfaces [3]. Makinde and Aziz et al. studied the nanofluids hydrodynamics of boundary layer flow on stretching surfaces. Sandeep et al. studied the effect of unsteady convective flow of nanofluids based on ethylene glycol on a vertical plate [5]. Akbar et al. studied the radiation effects on MHD flow of nanofluids on the stretching surfaces. Analytical solution of Casson nano fluids is optimized by Nadeem et al. [7]. Gireesha et al. [8] studied the MHD flow and characteristics of nano fluids and its heat transfer properties on stretching surfaces. by Zaimi et al. [9] studied the nanofluids and phenomena of boundary layer and heat transfer on a non linear stretching sheets. Mohankrishna et al. [10] discussed the effects of radiation on unsteady convective flow of nanofluids over a vertical plate as heat source. Akbar et al. [11], [12] discussed the flow of CNT Prandtl and suspended nano fluids on different sheets. Nadeem et al. [13] studied the partial slip effect on non-aligned stagnation flow on convective stretching surfaces. Malvandi et al. [14] 
studied the flow of nanofluids on the stretching surfaces slip effects was also considered. Prasad et al. [15] studied the variation in viscosity on MHD flow on stretching surfaces. Various studies are carried on dusty nanofluids which can be seen in literature [16-21]. Sheikholeslami et al. [22] studied the heat transfer and physical properties of nanofluids. Rashidi et al. [23] discussed the effect of buoyancy on MHD flow of nanofluids in radiations. Hayat et al.[24] studied the MHD flow of nanofluids on a stretching sheet. Sheikholeslami and Ganji [25] studied the effect of nanofluids in rotating frame. Elbashbeshy et al. [26] studied different properties of radiation on nanofluids on a moving surface with different thicknesses. Khader et al. [27] studied the boundary layer flow and slip boundary conditions. Abdel-Wahed et al. [28] simulated the effect of different thicknesses on the heat source of nanofluids. Hayat et al. [29] discussed the characteristics of different thicknesses on the behavior of flow and the parameters that are considered are temperature and velocity. The Brownian motion also plays an important role in nanofluids where real BC are imposed [30]. Rout and Mishra [31] studied the heat energy carrying on nanofluids on the stretching surfaces. Some more studies about these type of flows are presented in Refs. [3239].

Main purpose of this study is to analyze the flow of dust particles in hybrid nanofluid. In previous literature there is no study in Marangoni convective flow of hybrid nanofluid is accounted by considering silver and copper as nanoparticles and water as base fluid. Porous medium is placed over a stretching sheet. Flow is generated via stretching sheet. MHD effects are also considered. Effect of pertinent variables are studied in detail against velocity, temperature, Nusselt number and surface drag force. Also the present study is validated by comparing values of heat transfer with [32], [33] and [34].

\section{2: Modeling}


Two dimensional, steady MHD flow of hybrid nanofluid is considered. Marangoni convective boundary conditions are considered for momentum equation. Dust particles flow in hybrid nanofluid is examined in detail. Comparative results of dust phase and fluid phase are presented. Silver and $\mathrm{Cu}$ are nanoparticles and water is base fluid. Porous medium is considered. Cartesian coordinates are used where sheet is place along $x$ - axis and flow is towards positive $y$-axis. $u_{w}(x)$ is the stretching velocity of the sheet along $x$ - axis (See Fig. 1).

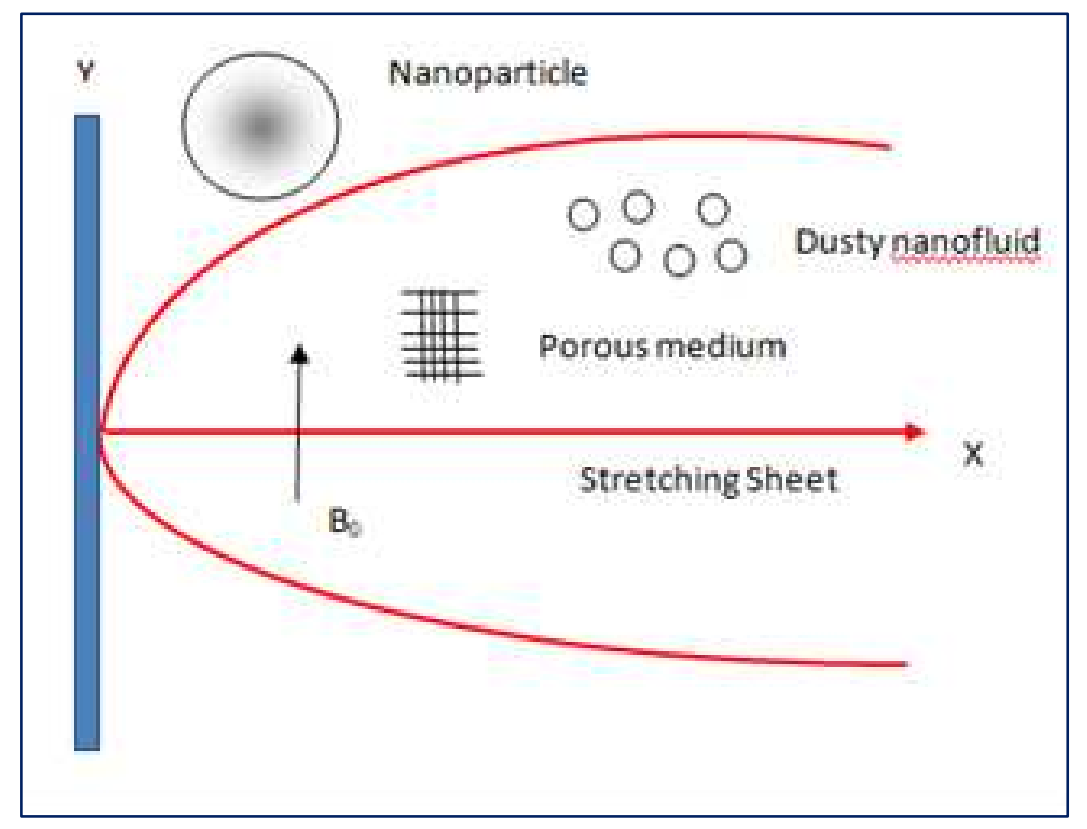

Fig. 1: Flow diagram

Required equations of dust phase and fluid phase are designed as [8], [32], [34] and [39]:

$$
\begin{aligned}
& \frac{\partial u}{\partial x}+\frac{\partial v}{\partial y}=0 \\
& \frac{\partial u_{p}}{\partial x}+\frac{\partial v_{p}}{\partial y}=0 \\
& \left(1-\phi_{p}\right)\left(u \frac{\partial u}{\partial x}+v \frac{\partial u}{\partial y}\right)=\left(1-\phi_{p}\right) v_{h n f} \frac{\partial^{2} u}{\partial y^{2}}+\frac{K N}{\rho_{h n f}}\left(u_{p}-u\right)-\frac{\sigma_{h n f} B_{0}^{2} u}{\rho_{h n f}}-\frac{v_{h n f}}{k_{1}} u
\end{aligned}
$$




$$
\left.\begin{array}{l}
u_{p} \frac{\partial u_{p}}{\partial x}+v_{p} \frac{\partial u_{p}}{\partial y}=\frac{K}{m}\left(u_{p}-u\right), \\
\left(\rho c_{p}\right)_{h n f}\left(u \frac{\partial T}{\partial x}+v \frac{\partial T}{\partial y}\right)=k_{h n f} \frac{\partial^{2} T}{\partial y^{2}}+\frac{N_{1}\left(c_{p}\right)_{h n f}}{\tau_{T}}\left(T_{p}-T\right)+\frac{N_{1}}{\tau_{v}}\left(u_{p}-u\right)^{2}, \\
N_{1} c_{m}\left(u_{p} \frac{\partial T_{p}}{\partial x}+v_{p} \frac{\partial T_{p}}{\partial y}\right)=-\frac{N_{1}\left(c_{p}\right)_{h n f}}{\tau_{T}}\left(T_{p}-T\right), \\
\mu_{h n f} \frac{\partial u}{\partial y}=\frac{d \sigma}{d T} \frac{d T}{d x}, v=0, T=T_{w}=T_{\infty}+A x^{2} \text { at } y=0, \\
u \rightarrow 0, u_{p} \rightarrow 0, \quad v_{p} \rightarrow v, T \rightarrow T_{\infty}, T_{p} \rightarrow T_{\infty} \text { at } y \rightarrow \infty,
\end{array}\right\}
$$

where $(u, v), T$ and $\left(u_{p}, v_{p}\right), T_{p}$ are fluid and dust phase velocity vectors and temperature respectively. The volume fraction of the nanoparticles (silver and copper) and dust particle are $\phi_{1}$, $\phi_{2}$ and $\phi_{p}$ respectively, $v, N, K, m, B_{0}, \mu_{h n f}, \rho_{h n f},\left(\rho c_{p}\right)_{h n f}, k_{h n f}, N_{1}=N m, \tau_{T}, \tau_{v}, c_{p}$, $c_{m}, k_{1}, T_{w}, T_{\infty}$ denote the kinematic viscosity, number density of dust particles, density, Stokes resistance, dust particle of mass, magnetic field strength, dynamic viscosity, density, heat capacitance of hybrid nanofluid, respectively, particles phase density, thermal equilibrium and relaxation time of dust particles, specific heat for fluid and dust particles, porosity constant, the porosity parameter, wall temperature and ambient temperature respectively. Surface tension is defined as $\sigma=\sigma_{0}\left(1-\gamma_{T}\left(T-T_{\infty}\right)\right)$ where $\gamma_{T}$ is positive fluid property.

Transformations are considered as:

$$
\left.\begin{array}{c}
u=\frac{c_{1}}{c_{2}} x f^{\prime}(\eta), \quad v=\frac{-1}{c_{2}} f(\eta), \eta=\frac{1}{c_{1}} y, \quad \theta=\frac{T-T_{\infty}}{A x^{2}}, \\
u_{p}=\frac{c_{1}}{c_{2}} x F^{\prime}(\eta), \quad v_{p}=\frac{-1}{c_{2}} F(\eta), \eta=\frac{1}{c_{1}} y, \quad \theta_{p}=\frac{T_{p}-T_{\infty}}{A x^{2}} .
\end{array}\right\}
$$

After implementation of Eq. (8), Eqs. (1) and (2) are satisfied and Eqs. (3), (4), (5), (6) and (7) 
take the form

$$
\begin{aligned}
& \frac{f^{\prime \prime \prime}}{A_{1} A_{2} \operatorname{Re}}+f f^{\prime \prime}-f^{\prime 2}+\frac{1}{A_{1}\left(1-\phi_{p}\right)} \alpha \beta\left(F^{\prime}-f^{\prime}\right)-\frac{\sigma_{h n f}}{\sigma_{f}} \frac{M}{A_{1}\left(1-\phi_{p}\right)} f^{\prime}-\frac{K^{*}}{A_{2} A_{1}\left(1-\phi_{p}\right)} f^{\prime}=0, \\
& F^{\prime 2}-F F^{\prime \prime}+\beta\left(F^{\prime}-f^{\prime}\right)=0, \\
& \frac{A_{4}}{A_{2} \operatorname{Pr}} \theta^{\prime \prime}-2 f^{\prime} \theta+f \theta^{\prime}+\frac{\alpha \beta_{T}}{A_{1}}\left(\theta_{p}-\theta\right)+E c \alpha \frac{1}{A_{2}}\left(F^{\prime}-f^{\prime}\right)^{2}=0, \\
& 2 F^{\prime} \theta_{p}-F^{\prime} \theta_{p}+\gamma \beta_{T}\left(\theta_{p}-\theta\right)=0, \\
& f^{\prime \prime}(0)=-2 A_{2}, f(0)=0, \quad \theta(0)=1, \quad \text { at } \eta=0, \\
& \left.f^{\prime}(\eta)=0, F^{\prime}(\eta)=0, \quad F(\eta)=f(\eta), \quad \theta(\eta)=0, \theta_{p}(\eta)=0, \text { at } \eta \rightarrow \infty,\right\}
\end{aligned}
$$

where Re, $\alpha, \beta, M, K^{*}, \operatorname{Pr}, \beta_{T}, \gamma$ and $E c$ define Reynolds number, dust particles with mass concentration, particles interaction parameter, magnetic parameter, porosity parameter, Prandtl number, interaction parameter, specific heat ratio and Eckert number.

$$
\left.\begin{array}{c}
\operatorname{Re}\left(=\frac{v_{f}}{c_{1} c_{2}}\right), \alpha\left(=\frac{N m}{\rho_{b f}}\right), \beta\left(=\frac{K c_{2}}{m c_{1}}\right), M\left(=\frac{\sigma_{f} B_{0}^{2} c_{2}}{\rho_{b f} c_{1}}\right), \operatorname{Pr}\left(=\frac{k_{f} c_{2} c_{1}}{\left(\rho c_{p}\right)_{b f}}\right), \\
\beta_{T}\left(=\frac{c_{2}}{c_{1} \tau_{T}}\right), \gamma\left(=\frac{\left(c_{p}\right)_{h m f}}{c_{m}}\right), E c\left(=\frac{c_{1}}{c_{2} c_{p} A \tau_{v}}\right)
\end{array}\right\}
$$

Nusselt number and skin friction for given flow system are defined as

$$
\left.\begin{array}{c}
N u_{x}=\frac{x q_{w}}{k_{f} A x^{2}}, \\
C f_{x}=\frac{\tau_{w}}{\rho_{f}\left(\frac{1}{c_{2}}\right)^{2}},
\end{array}\right\}
$$

where 


$$
\left.\begin{array}{c}
q_{w}=-\left.k_{h n f} \frac{\partial T}{\partial y}\right|_{y=0}, \\
\tau_{w}=\left.\mu_{h n f} \frac{\partial u}{\partial y}\right|_{y=0},
\end{array}\right\}
$$

so dimensionless forms are

$$
\left.\begin{array}{c}
N u_{x} \operatorname{Re}^{-0.5}=-A_{4} \theta^{\prime}(0), \\
C f_{x} \operatorname{Re}^{0.5}=\frac{1}{A_{2}} f^{\prime \prime}(0),
\end{array}\right\}
$$

with $\operatorname{Re}=c_{1}^{2} x^{2}$ Reynolds number and $A_{1}, A_{2}, A_{3}, A_{4}$ and $A_{5}$ are expressed as:

Table 1: Mathematical form of thermo-physical properties.

\begin{tabular}{|l|l|}
\hline & $A g-C u-H_{2} O$ hybrid nanofluid \\
\hline Density $\left(A_{1}\right)$ & $\frac{\rho_{h n f}}{\rho_{f}}=\left(1-\phi_{1}\right)\left[\left(1-\phi_{1}\right)+\phi_{1} \frac{\rho_{s 1}}{\rho_{f}}\right]+\phi_{2} \frac{\rho_{s 2}}{\rho_{f}}$, \\
\hline heat capacity $\left(A_{3}\right)$ & $\frac{\left(\rho c_{p}\right)_{h n f}}{\left(\rho c_{p}\right)_{f}}=\left(1-\phi_{2}\right)\left[\left(1-\phi_{1}\right)+\phi_{1} \frac{\left(\rho c_{p}\right)_{s 1}}{\left(\rho c_{p}\right)_{f}}\right]+\phi_{2} \frac{\left(\rho c_{p}\right)_{s 2}}{\left(\rho c_{p}\right)_{f}}$, \\
\hline viscosity $\left(A_{1}\right)$ & $\mu_{h n f}=\frac{\mu_{f}}{\left(1-\phi_{1}\right)^{2.5}\left(1-\phi_{2}\right)^{2.5}}$, \\
\hline thermal conductivity $\left(A_{4}=\frac{k_{h n f}}{k_{f}}\right)$ & $\frac{k_{h n f}}{k_{b f}}=\frac{k_{s 2}+2 k_{b f}-2 \phi_{2}\left(k_{b f}-k_{s 2}\right)}{k_{s 2}+2 k_{b f}+\phi_{2}\left(k_{b f}-k_{s 2}\right)}, \frac{k_{b f}}{k_{f}}=\frac{k_{s 1}+2 k_{f}-2 \phi_{1}\left(k_{f}-k_{s 1}\right)}{k_{s 1}+2 k_{f}+\phi_{1}\left(k_{f}-k_{s 1}\right)}$, \\
\hline electrical conductivity $\left(A_{5}=\frac{\sigma_{h n f}}{\sigma_{f}}\right)$ & $\frac{\sigma_{h n f}}{\sigma_{b f}}=\frac{\sigma_{s 2}+2 \sigma_{b f}-2 \phi_{2}\left(\sigma_{b f}-\sigma_{s 2}\right)}{\sigma_{s 2}+2 \sigma_{b f}+\phi_{2}\left(\sigma_{b f}-\sigma_{s 2}\right)}, \frac{\sigma_{b f}}{\sigma_{f}}=\frac{\sigma_{s_{1}}+2 \sigma_{f}-2 \phi_{1}\left(\sigma_{f}-\sigma_{s 1}\right)}{\sigma_{s 1}+2 \sigma_{f}+\phi_{1}\left(\sigma_{f}-\sigma_{s 1}\right)}$, \\
\hline Prandtl number & 6.2 \\
\hline
\end{tabular}

Table 1 highlights the mathematical form of transport quantities. Numerical values of thermophysical properties of materials involved in present study are presented in Table 2.

Table 2: Numerical values of thermo-physical properties of silver, copper and water. 


\begin{tabular}{|l|l|l|l|l|l|}
\hline & $k(\mathrm{~W} / \mathrm{mk})$ & $\rho\left(\mathrm{kg} / \mathrm{m}^{3}\right)$ & $\sigma(\mathrm{Um})^{-1}$ & $c_{p}(\mathrm{~J} / \mathrm{kgk})$ & $\rho c_{p}\left(j / \mathrm{m}^{3} \mathrm{k}\right)$ \\
\hline$(\mathrm{Ag})$ & 429 & 10490 & $6.30 \times 10^{7}$ & 235 & 2465150 \\
\hline$(\mathrm{Cu})$ & 401 & 8933 & $5.96 \times 10^{7}$ & 385 & 3439205 \\
\hline$\left(\mathrm{H}_{2} \mathrm{O}\right)$ & 0.613 & 997.1 & 0.05 & 4179 & 4166880.9 \\
\hline
\end{tabular}

\section{3: Discussion}

Figs. (2-13) is made to show the trend and behavior of involved parameters against velocity and temperature of $\mathrm{Ag}-\mathrm{Cu}-\mathrm{H}_{2} \mathrm{O}$ hybrid nanofluid and dust particles. Comparative results of both fluid phase and dust phase are presented through graphs. Table 2 shows the results of skin friction and Nusselt number via various variables.

Fig. 2 describes the characteristics of hybrid nanofluid $\left(\mathrm{Ag}-\mathrm{Cu}-\mathrm{H}_{2} \mathrm{O}\right)$ and dusty fluid phase against magnetic parameter $(M)$. It is witnessed that velocities $\left(f^{\prime}, F^{\prime}\right)$ for both phases decay with increase in magnetic parameter. Reason behind decreasing trend is that $(M=0,0.7,1.4,2.1)$ is directly proportional to Lorentz force which produces resistance between the particles to flow hence velocity decays. Furthermore it is also seen that decrease in dust phase is more as compared to hybrid nanofluid because dust particles are denser than nanoparticles. Due to this reason velocity of dust particle is less than nanoparticles. Fig. 3 tells the impact of mass concentration of dust particles $(\alpha)$ against fluid phase. We can see that fluid velocity enhances for greater values of $(\alpha=0,1,2,3)$. Since density of base fluid is inversely proportional to $(\alpha)$ so due to less density velocity of the hybrid nanofluid enhances. Influence of interaction parameter $(\beta)$ on fluid phase and dust phase is presented in Fig. 4. Decrease in $\left(f^{\prime}, F^{\prime}\right)$ is seen for higher estimation of 
$(\beta=0,1,2,3)$. Impact of volume fraction of dust particles on hybrid nanofluid flow is seen in Fig.

5. Increment in velocity of hybrid nanofluid is seen for greater estimation of $\left(\phi_{p}=0,0.2,0.4,0.6\right)$.

Physically we can say that with increase in $\phi_{p}$ density of dust particles enhances due to which velocity decays. Porosity parameter effect on velocity of hybrid nanofluid $\left(f^{\prime}\right)$ and dust nanofluid $\left(F^{\prime}\right)$ in Fig. 6. It is seen that motion of the both fluids decay against higher $\left(K^{*}=0,0.4,0.8,1.2\right)$. Viscosity of the fluid rises due to increase in $\left(K^{*}=0,0.4,0.8,1.2\right)$ so resistance of the fluid particles also enhances consequently $\left(f^{\prime}, F^{\prime}\right)$ decay. Figs. 7 and 8 display the effect of nanoparticle volume fraction of silver and copper nanoparticles $\left(\left(\phi_{1}\right),\left(\phi_{2}\right)\right)$ on both the velocities. There is reducing effect is noticed via larger $\left(\phi_{1}=0,0.1,0.2,0.3\right)$ and $\left(\phi_{2}=0,0.1,0.2,0.3\right)$. Physically motion of the fluids slow down because when there is more saturation of nanoparticles in the fluid than nanofluid will be more dense due which velocity reduces. It is also observed that velocity in fluid phase is more than dust phase. Impact of interaction parameter $\left(\beta_{T}\right)$ on temperature field of hybrid nanofluid and dusty nanofluid is portrayed in Fig. 9. It is seen that temperatures of both fluids $\left(\theta, \theta_{p}\right)$ decays for higher $\left(\beta_{T}=0,0.3,0.6,0.9\right)$. Physically surface tension decays with increase in $\left(\beta_{T}\right)$ due to which resistance between the fluid particles also reduces consequently $\left(\theta, \theta_{p}\right)$ decay. It is worthmentioning that temperature for hybrid nanofluid $(\theta)$ is more as compared to dusty nanofluid $\left(\theta_{p}\right)$. Specific heat ratio effect $(\gamma)$ on $\left(\theta_{p}\right)$ is seen in Fig. 10 By definition of $\gamma$ we can say that specific heat of dust particles decays by larger $\gamma$. So physically when $c_{m}$ reduces the amount of heat required by the unit mass also decays due to which temperature decay. Impact of $\phi_{p}$ against temperature of dust fluid phase is exhibited in Fig. 11. It 
is witnessed that values of temperature are decreasing via larger $\left(\phi_{p}=0.1,0.8,0.87,0.9\right)$. Variation of temperature profile of dust fluid phase and hybrid nanofluid phase against $\phi_{2}$ is seen through Fig. 12. It is shown that temperature of dust fluid phase is reducing and for hybrid nanofluid temperature is increasing. Physically when we increase the values of $\left(\phi_{2}=0,0.2,0.4,0.6\right)$ nanoparticles in hybrid nanofluid enhances due to which more resistance is produced and temperature $(\theta)$ rises. Fig. 13 elaborates the impact of magnetic parameter against temperature profile $(\theta)$. Physically when $M$ increases there is more resistance development between the fluid particles due to which $\theta$ rises. Table 3 is designed to show the variation of skin friction and Nusselt number against pertinent parameters via hybrid nanofluid. Here we witnessed that surface drag force decays for $\alpha, \phi_{1}$ and $\beta_{T}$ and opposite behavior is seen for $\phi_{p}, M$ and $\beta$. Heat transfer rate have inverse relation with $\phi_{1}, \phi_{p}$ and $\beta_{T}$ while increase in Nusselt number is reported for higher $\alpha, M$ and $\beta$. Table 4 is designed for validation of our problem in particular case with [32], [33] and [34]. It is noted that there is approximately equal values of $-\theta^{\prime}(0)$ in present study with previous literature.

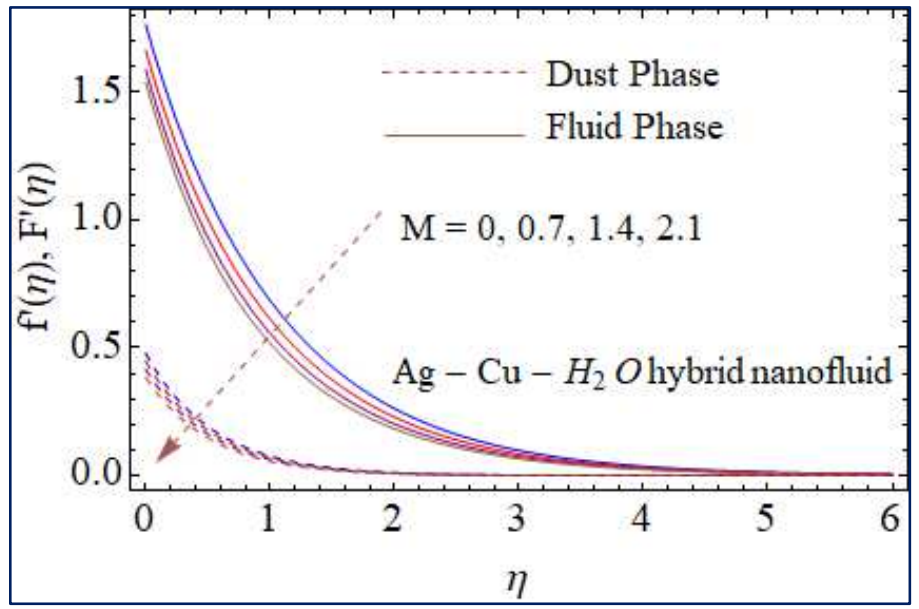

Fig. 2: $f^{\prime}(\eta)$ and $F^{\prime}(\eta)$ versus $M$. 


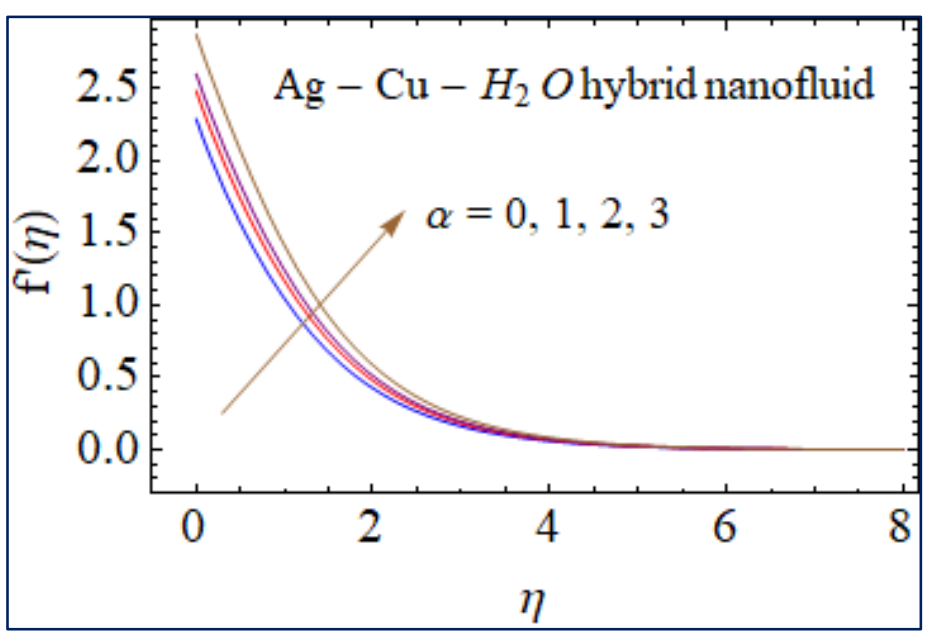

Fig. 3: $f^{\prime}(\eta)$ versus $\alpha$.

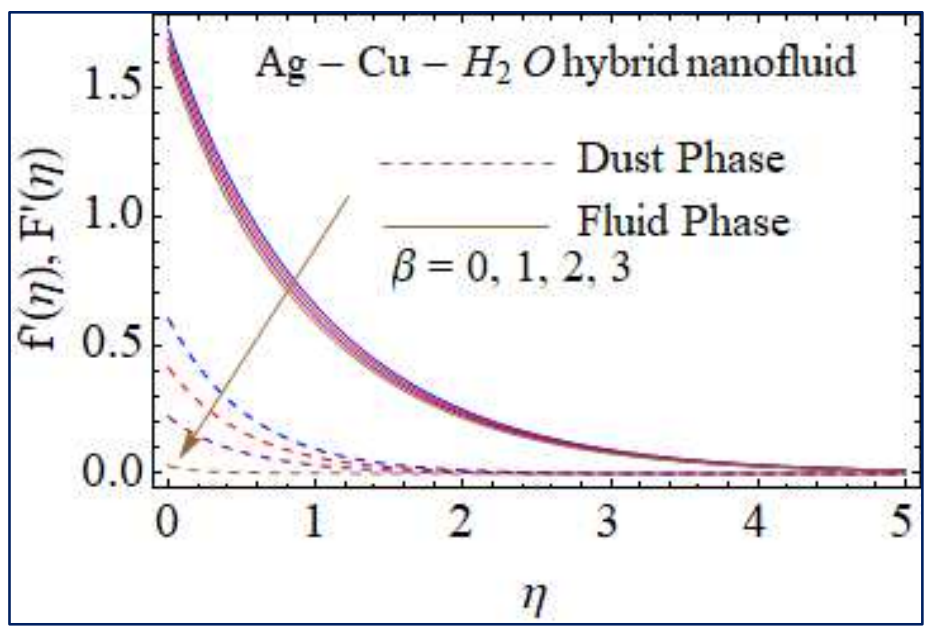

Fig. $4: f^{\prime}(\eta)$ and $F^{\prime}(\eta)$ versus $\beta$.

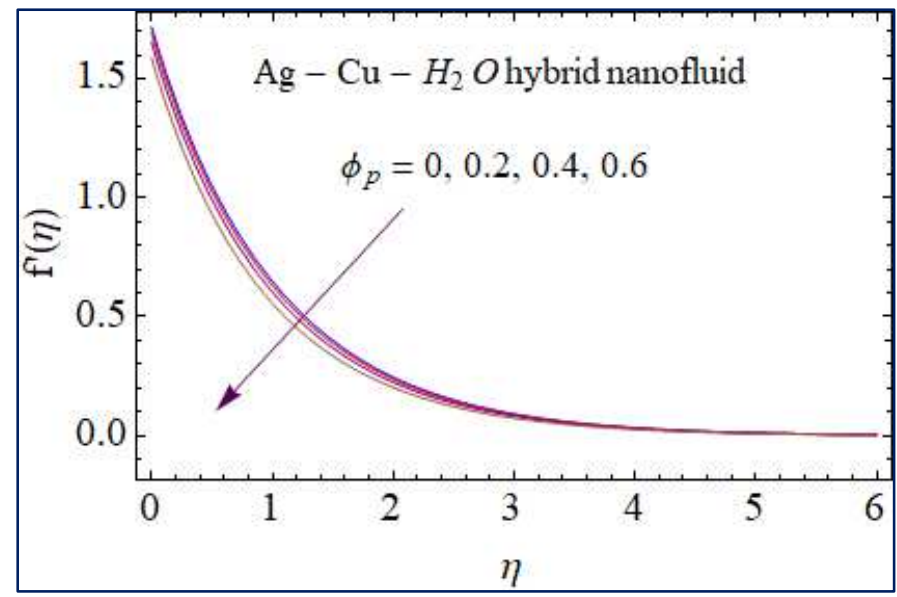

Fig. 5: $f^{\prime}(\eta)$ versus $\phi_{p}$. 


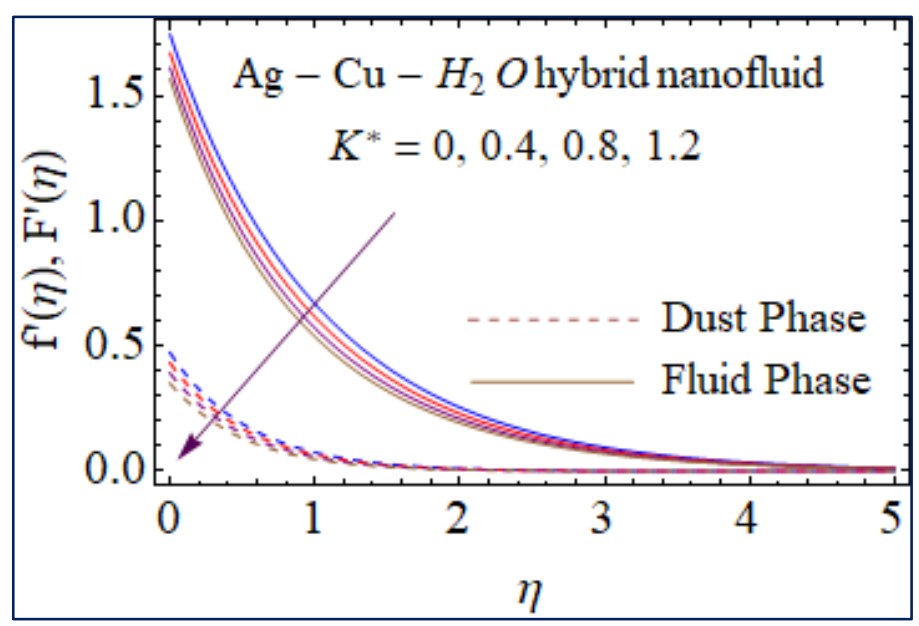

Fig. 6: $f^{\prime}(\eta)$ and $F^{\prime}(\eta)$ versus $K^{*}$.

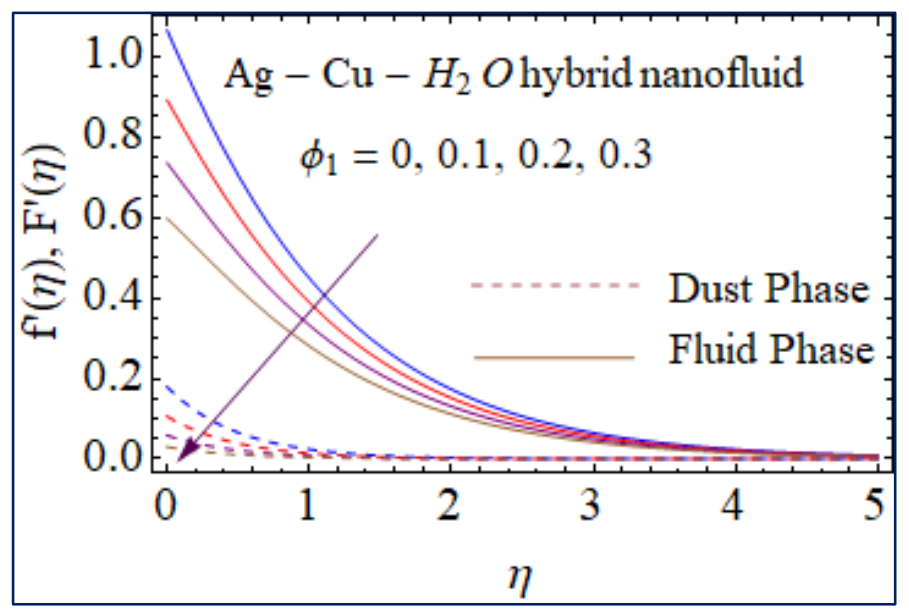

Fig. 7: $f^{\prime}(\eta)$ and $F^{\prime}(\eta)$ versus $\phi_{1}$.

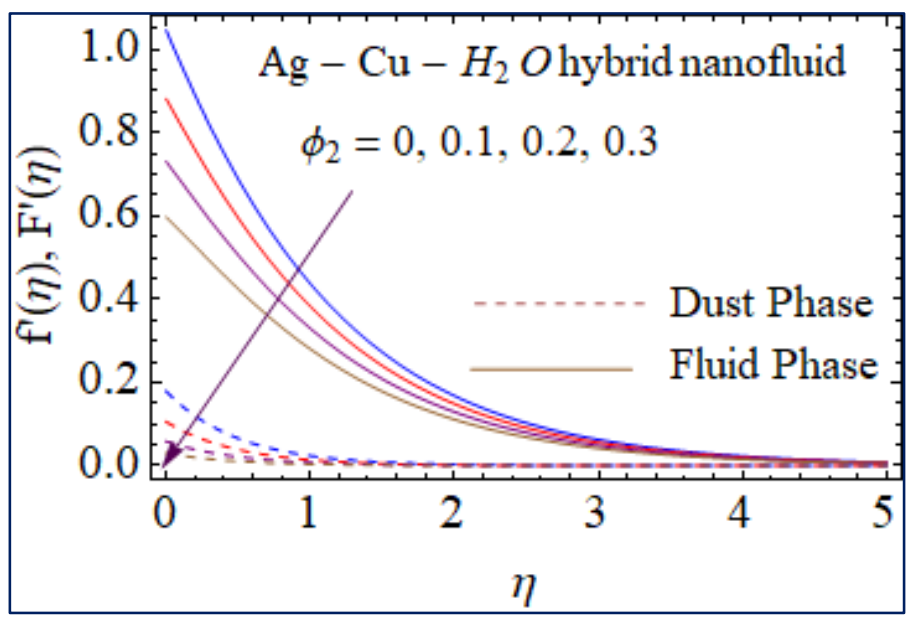

Fig. 8: $f^{\prime}(\eta)$ and $F^{\prime}(\eta)$ versus $\phi_{2}$. 


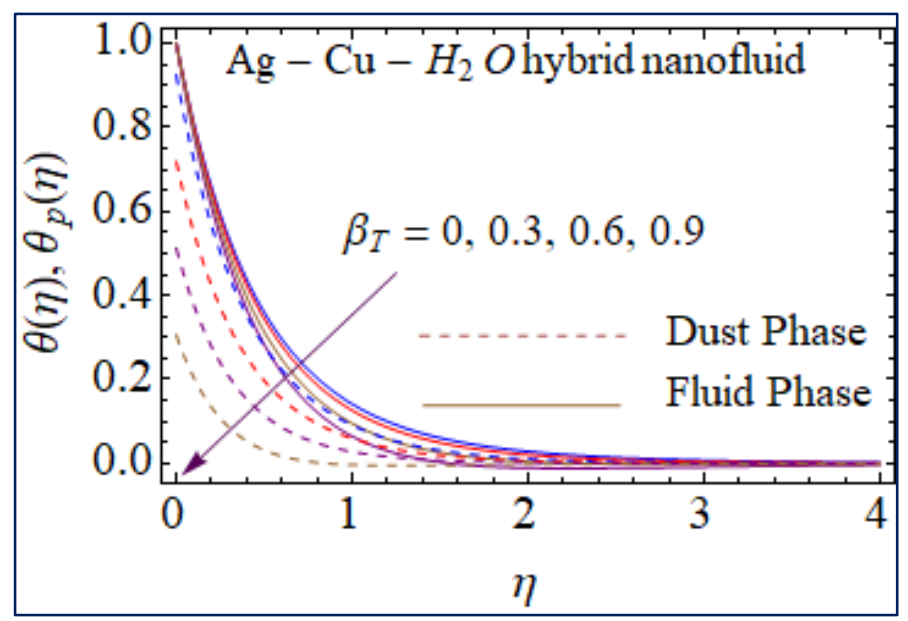

Fig. 9: $\theta(\eta)$ and $\theta_{p}(\eta)$ versus $\beta_{T}$.

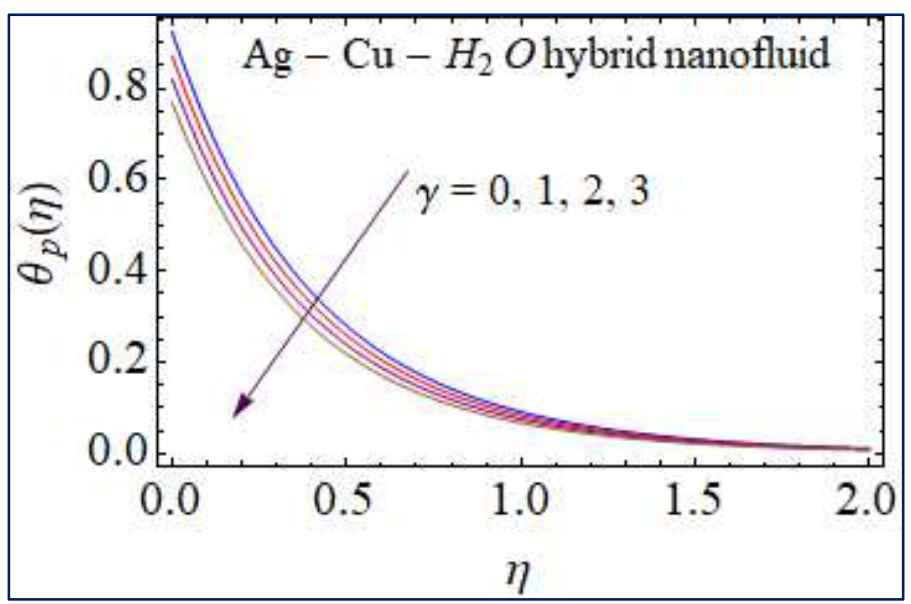

Fig. 10: $\theta_{p}(\eta)$ versus $\gamma$.

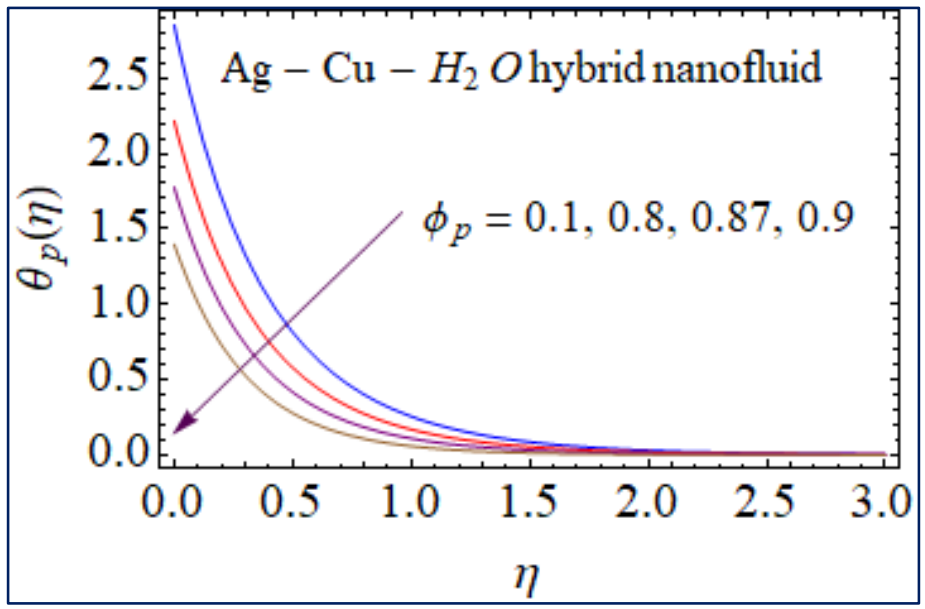

Fig. 11: $\theta_{p}(\eta)$ versus $\phi_{p}$. 


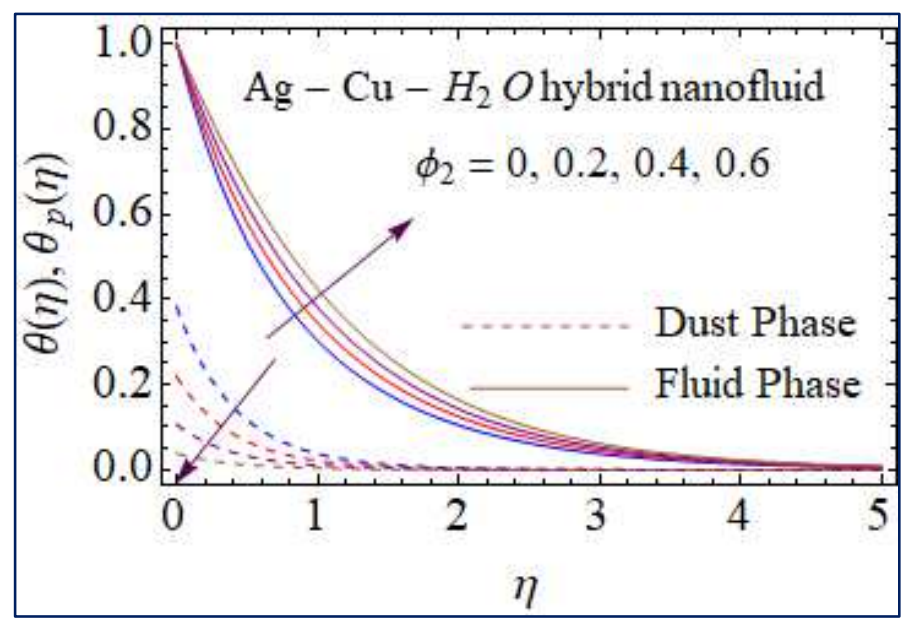

Fig. 12: $\theta(\eta)$ and $\theta_{p}(\eta)$ versus $\phi_{2}$.

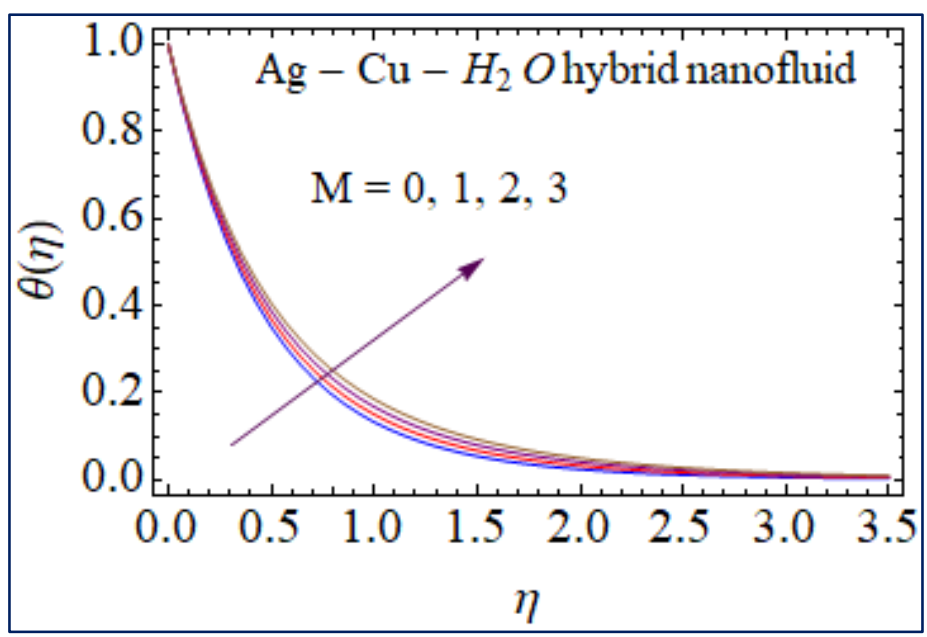

Fig. 13: $\theta(\eta)$ versus $M$.

Table 3: Numerical values of Nusselt number and skin friction.

\begin{tabular}{|l|l|l|l|l|l|l|l|}
\hline & & & & & & \multicolumn{2}{|c|}{$\mathrm{Ag}-\mathrm{Cu}-\mathrm{H}_{2} \mathrm{O}$ hybrid nanofluid } \\
\hline$\phi_{1}$ & $\phi_{p}$ & $M$ & $\alpha$ & $\beta$ & $\beta_{T}$ & $A_{2} f^{\prime \prime}(0)$ & $-A_{4} \theta^{\prime}(0)$ \\
\hline 0 & 0.2 & 0.4 & 0.4 & 0.2 & 0.3 & -0.65536 & 0.045264 \\
\hline 0.1 & & & & & & -0.38698 & 0.021545 \\
\hline 0.1 & 0.3 & & & & & -0.75520 & 0.004565 \\
\hline & 0.4 & & & & & -0.95520 & 0.000314 \\
\hline
\end{tabular}




\begin{tabular}{|l|l|l|l|l|l|l|}
\hline 0.2 & 0.5 & & & & -0.74545 & 0.124522 \\
\hline & 0.6 & & & & -0.82125 & 0.325446 \\
\hline & 0.4 & 0.6 & & & -0.52354 & 0.141245 \\
\hline & & 0.8 & & & -0.35461 & 0.215456 \\
\hline & & 0.4 & 0.3 & & -0.65811 & 0.215746 \\
\hline & & & 0.4 & & -0.68412 & 0.431578 \\
\hline & & & 0.2 & 0.4 & -0.56287 & 0.0012452 \\
\hline & & & & 0.5 & -0.55214 & 0.0001236 \\
\hline
\end{tabular}

Table 4: Comparative values of $-\theta^{\prime}(0)$ with [32], [33] and [34] when $E c=\beta_{T}=\beta=\alpha=\phi_{1}=\phi_{2}=\phi_{p}=0, \operatorname{Re}=1$ and $f^{\prime \prime}(0)=-2 A_{2}$ replaces by $f^{\prime}(0)=1$.

\begin{tabular}{|l|l|l|l|l|}
\hline Pr & Sandeep et al. [32] & Abel et al. [33] & Gireesha et al. [34] & Our results \\
\hline 0.72 & 1.08861 & 1.08865 & 1.0886 & 1.0885623 \\
\hline 1 & 1.333333 & 1.3333 & 1.3333 & 1.333333 \\
\hline 10 & 4.796817 & 4.7968 & 4.7968 & 4.796819 \\
\hline
\end{tabular}

\section{4: Conclusions}

Main results of hybrid nanofluid and dust fluid phase flow with Marangoni convection are presented below:

- Velocities $f^{\prime}$ and $F^{\prime}$ reduces for $M, \beta, K^{*}, \phi_{1}$ and $\phi_{2}$.

- Velocity of hybrid nanofluid is more as compared to dust fluid phase.

- Temperatures $\theta$ and $\theta_{p}$ decay for larger $\left(\beta_{T}\right)$. 
- Temperature of hybrid fluid is more as compared to $\theta_{p}$.

- Hybrid nanofluid flow enhances for greater $\left(\phi_{2}\right)$ while there is decrease in $\theta_{p}$ with increase in $\left(\phi_{2}\right)$.

\section{References}

[1] S. U. S. Choi, Enhanced thermal conductivity of nanofluids with nano particles, development and applications of Newtonian flows, FED vol. 231/MD-vol.66 (1995) 99--105.

[2] F. E. Marble, Dynamics of dusty gases, Annu. Rev. Fluid Mech. 2 (1970) 397--446, doi:10.1146/annurev.fl.02.010170.002145.

[3] A. Chakrabarti, A.S. Gupta, Hydromagnetic flow and heat transfer over a stretching sheet, Q. Appl. Math. 37 (1979) 73--98.

[4] O. D. Makinde, A. Aziz, Boundary layer flow of a nanofluid past a stretching sheet with convective boundary condition, Int. J. Therm. Sci. 50 (2011) 1326-- 1332.

[5] N. Sandeep, V. Sugunamma, P. Mohankrishna, Effects of radiation on an unsteady natural convective flow of a EG-Nimonic 80a nanofluid past an infinite vertical plate, Adv. Phys. Theories Appl. 23 (2013) 36--43.

[6] N. S. Akbar, S. Nadeem, R. U. Haq, Z. H. Khan, Radiation effects on MHD stagnation point flow of nano fluid towards a stretching surface with convective boundary condition, Chin. J. Aeronaut. 26 (6) (2013) 1389--1397.

[7] S. Nadeem, R. Mehmood, N.S. Akbar, Optimized analytical solution for oblique flow of a Casson-nano fluid with convective boundary conditions, Int. J. Therm. Sci. 78 (2014) 90--100.

[8] B. J. Gireesha, G.S. Roopa, H.J. Lokesh, C.S. Bagewadi, MHD flow and heat transfer of a dusty fluid over a stretching sheet, Int. J. Phys. Math. Sci. 3 (1) (2012) 171--180.

[9] K. Zaimi, I. Ishak, I. Pop, Boundary layer flow and heat transfer over a nonlinearly permeable 
stretching/shrinking sheet in a nanofluid, Sci. Rep. 4 (2014) 4404, doi:10.1038/srep04404.

[10] P. Mohankrishna, V. Sugunamma, N. Sandeep, Radiation and magneticfield effects on unsteady natural convection flow of a nanofluid past an infinite vertical plate with heat source, Chem. Process Eng. Res. 25 (2014) 39--52.

[11] N. S. Akbar, Z. H. Khan, S. Nadeem, The combined effects of slip and convective boundary conditions on stagnation-point flow of CNT suspended nanofluid over a stretching sheet, J. Mol. Liquids 196 (2014) 21--25.

[12] N. S. Akbar, Z. H. Khan, R. U. Haq, S. Nadeem, Dual solutions in MHD stagnationpoint flow of a Prandtl fluid impinging on a shrinking sheet, Appl. Math. Mech. 35 (7) (2014) 813$-820$.

[13] S. Nadeem, R. Mehmood, N.S. Akbar, Partial slip effect on non-aligned stagnation point nanofluid over a stretching convective surface, J. Magn. Magn. Mater. 378 (2015) 457--462.

[14] A. Malvandi, F. Hedayati, D.D. Ganji, Slip effects on unsteady stagnation point flow of a nanofluid over a stretching sheet, Powder Technol. 253 (2014) 377--384.

[15] K.V. Prasad, D. Pal, V. Umesh, N.S.P. Rao, The effect of variable viscosity on MHD viscoelastic fluid flow and heat transfer over a stretching sheet, Commun. Nonlinear Sci. Numer. Simul. 15 (2010) 331--344.

[16] M. Turkyilmazoglu, Exact analytical solutions for heat and mass transfer of MHD slip flow in nanofluids. Chem. Eng. Sci. 84 (2012) 182--187.

[17] O. D. Makinde, W. A. Khan, Z. H. Khan, Buoyancy effects on MHD stagnation point flow and heat transfer of a nanofluid past a convectively heated stretching/shrinking sheet. Int. J. Heat Mass Transf. 62 (2013) 526--533.

[18] M. I. Khan, M. Waqas, T. Hayat and A. Alsaedi, A comparative study of Casson fluid with 
homogeneous-heterogeneous reactions, J. Colloid Interface Sci., 498 (2017) 85-90.

[19] M. I. Khan, F. Alzahrani, A. Hobiny and Z. Ali, Estimation of entropy optimization in Darcy-Forchheimer flow of Carreau-Yasuda fluid (non-Newtonian) with first order velocity slip, Alex. Eng. J., 59 (2020) 3953-3962.

[20] T. Hayat, M. Farooq, A. Alsaedi, Thermal radiation effect in MHD flow of Powell-- Eyring nanofluid induced by a stretching cylinder. Open Phys. 13 (2015) 188--197.

[21] M. Sheikholeslami, M. I. Khan, Y. M. Chu, S. Kadry and W. A. Khan, CVFEM based numerical investigation and mathematical modeling of surface dependent magnetized copperoxide nanofluid flow using new model of porous space, Numer. Meth. Partial Diff. Equat., In Press (2020).

[22] M. Sheikholeslami, M. Gorji-Bandpy, D. D. Ganji, Lattice Boltzmann method for MHD natural convection heat transfer using nanofluid. Powder Technol. 254 (2014) 82--93.

[23] M. M. Rashidi, N. V. Ganesh, A. K. A. Hakeem, B. Ganga, Buoyancy effect on MHD flow of nanofluid over a stretching sheet in the presence of thermal radiation. J. Mol. Liq. 198 (2014) 2348.

[24] T. Hayat, I. Ullah, T. Muhammad, A. Alsaedi, Magnetohydrodynamic (MHD) threedimensional flow of second grade nanofluid by a convectively heated exponentially stretching surface. J. Mol. Liq. 220 (2016) 1004--1012.

[25] M. Sheikholeslami, D. D. Ganji, Numerical investigation for two phase modeling of nanofluid in a rotating system with permeable sheet. J. Mol. Liq. 194 (2014) 13--19.

[26] E. M. A. Elbashbeshy, T. G. Emam, M. S. Abde-wahed, Flow and heat transfer over a moving surface with non-linear velocity and variable thickness in a nanofluid in the presence of thermal radiation. Can. J. Phys. 91 (2013) 699--708. 
[27] M. M. Khader, A. M. Megahed, Numerical solution for boundary layer flow due to a nonlinearly stretching sheet with variable thickness and slip velocity, Eur.Phys. J. Plus 128 (2013) 100.

[28] M. S. Abdel-Wahed, E. M. A. Elbashbeshy, T. G. Emam, Flow and heat transfer over a moving surface with non linear velocity and variable thickness in a nanofluids in the presence of Brownian motion. Appl. Math. Comput. 254 (2015) 49--62.

[29] T. Hayat, M. Farooq, A. Alsaedi, F. Al-Solamy, Impact of Cattaneo--Christov heat flux in the flow over a stretching sheet with variable thickness. AIP Adv. 5 (2015) 087159.

[30] A. V. Kuznetsov, D. A. Nield, Natural convective boundary-layer flow of a nanofluid past a vertical plate: a revised model. Int. J. Therm. Sci. 77 (2014) 126--129.

[31] B. C. Rout, S. R. Mishra, Thermal energy transport on MHD nanofluid flow over a stretchingsurface: A comparative study, Eng. Sci. Tech. Int. J. (2018) https://doi.org/10.1016/j.jestch.2018.02.007

[32] S. Naramgari, C. Sulochana, MHD flow of dusty nanofluid over a stretching surface with volume fraction of dusty particles, Ain Shams Eng. J. 7 (2) (2016) 709-716.

[33] M. S. Abel, N. Mahesha, Heat transfer in MHD viscoelastic fluid flow over a stretching sheet with variable thermal conductivity, non-uniform heat source and radiation, Appl. Math Model, 32 (2008) 1965-1983.

[34] B. J. Gireesha, G. S. Roopa, H. J. Lokesh, C. S. Bagewadi, MHD flow and heat transfer of a dusty fluid over a stretching sheet, Int. J. Phys. Math Sci., 3 (1) (2012) 171-180

[35] Y. M. Chu, M. Nazeer, M. I. Khan and F. Hussain, H. Rafi, S. Qayyum, Z. Abdelmalekhi, Combined impacts of heat source/sink, radiative heat flux, temperature dependent thermal conductivity on forced convective Rabinowitsch fluid, Int. Commun. Heat Mass Transf. (2020) 
105011 (In Press).

[36] M. Nazeer, M. I. Khan, M. U. Rafiq, N. B. Khan, Numerical and scale analysis of EyringPowell nanofluid towards a magnetized stretched Riga surface with entropy generation and internal resistance, Int. Commun. Heat Mass Transf. 119 (2020) 10496.

[37] M. K. Nayak, S. Shaw, M. I. Khan, V. S. Pandey and M. Nazeer, Flow and thermal analysis on Darcy-Forchheimer flow of copper-water nanofluid due to a rotating disk: A static and dynamic approach, J. Mat. Research Tech. 9 (4) (2020) 7387-7408.

[38] M. Nazeer, N. Ali, F. Ahmad, W. Ali, Adila Saleem, Z. Ali and A.Sarfraz, Effects of radiative heat flux and joule heating on electro-osmotically flow of non-Newtonian fluid: Analytical approach, Int. Commun. Heat Mass Transf. 117 (2020) 104744.

[39] R. Naz, F. Mabood and T. Hayat, Inclined magnetic field effects on Marangoni flow of Carreau liquid, Thermal Sci. 24 (2020) 1131-1141.

\section{Author Contribution statement}

The research work is done completely by M. Ijaz Khan. 
Figures

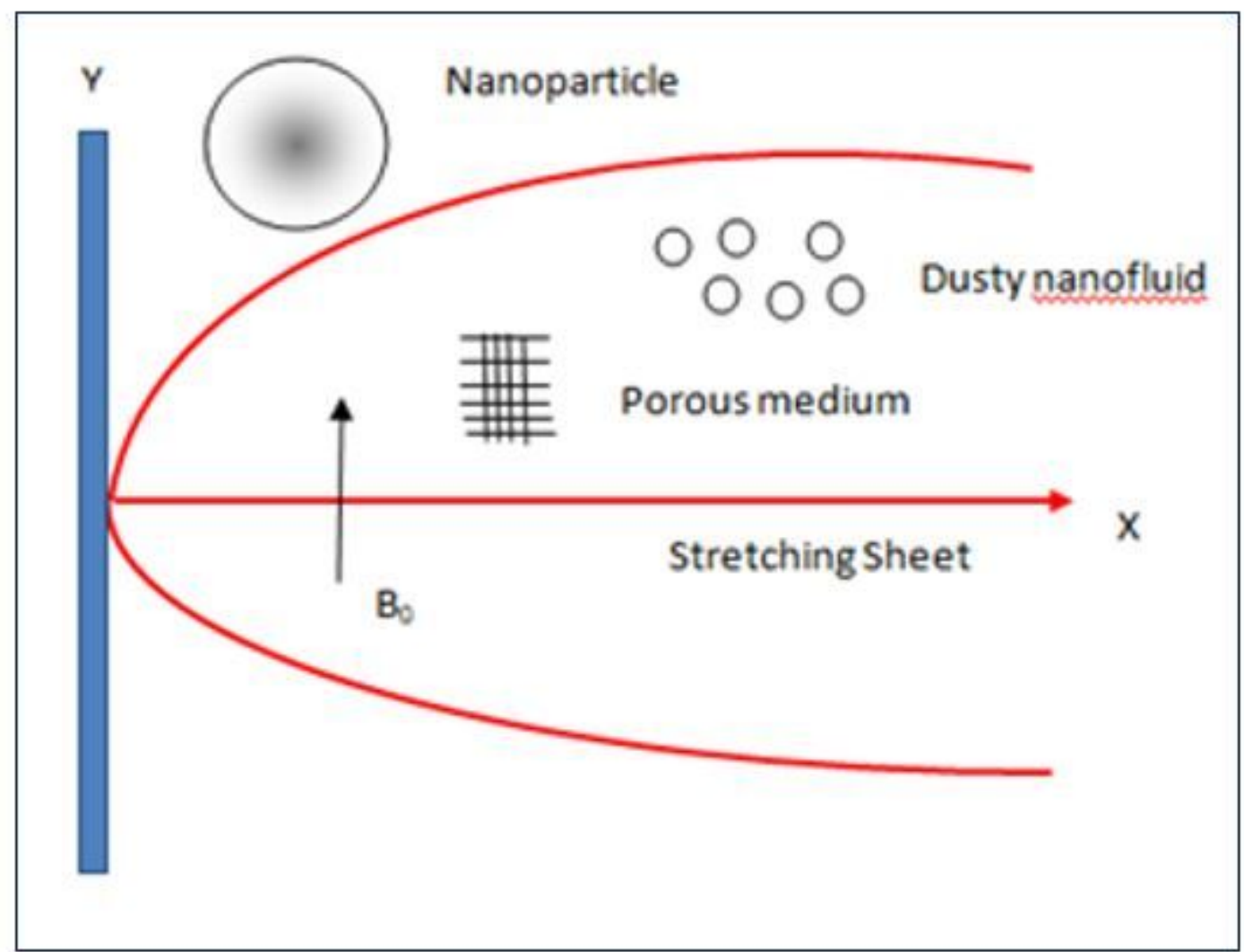

Figure 1

Flow diagram

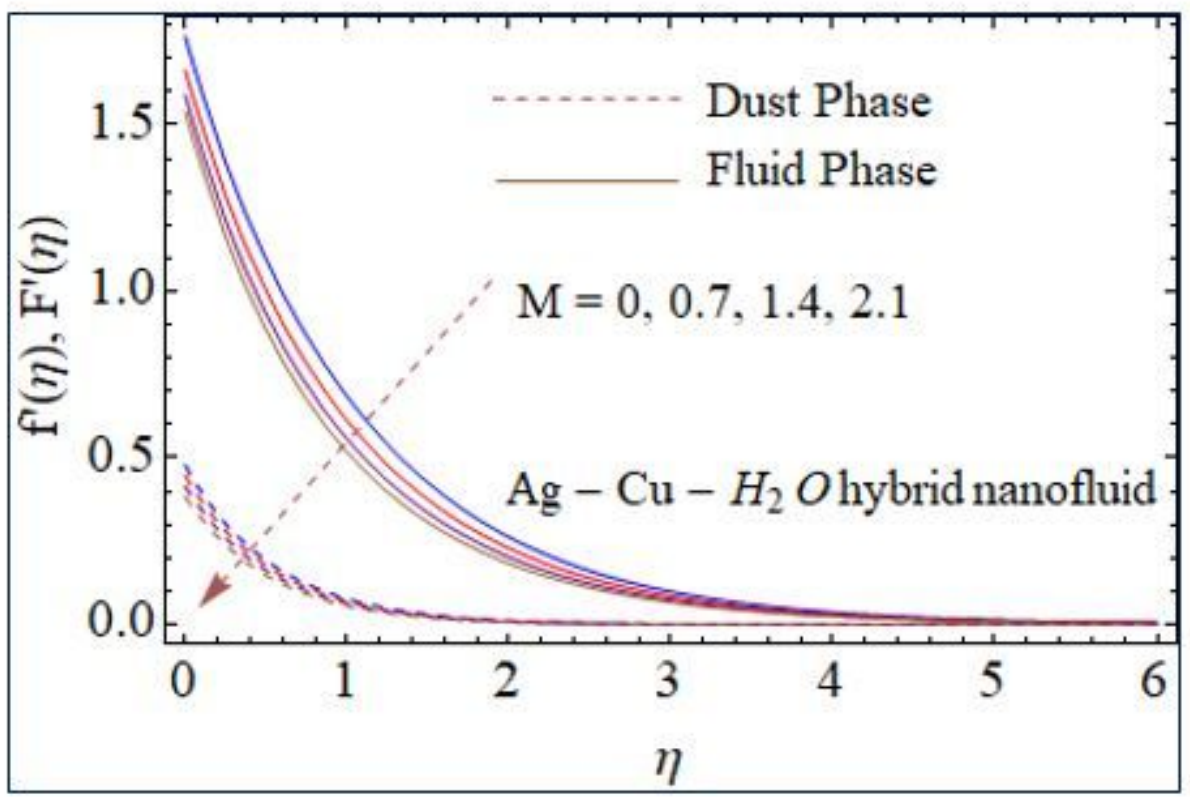

Figure 2 
please see the manuscript file for the full caption

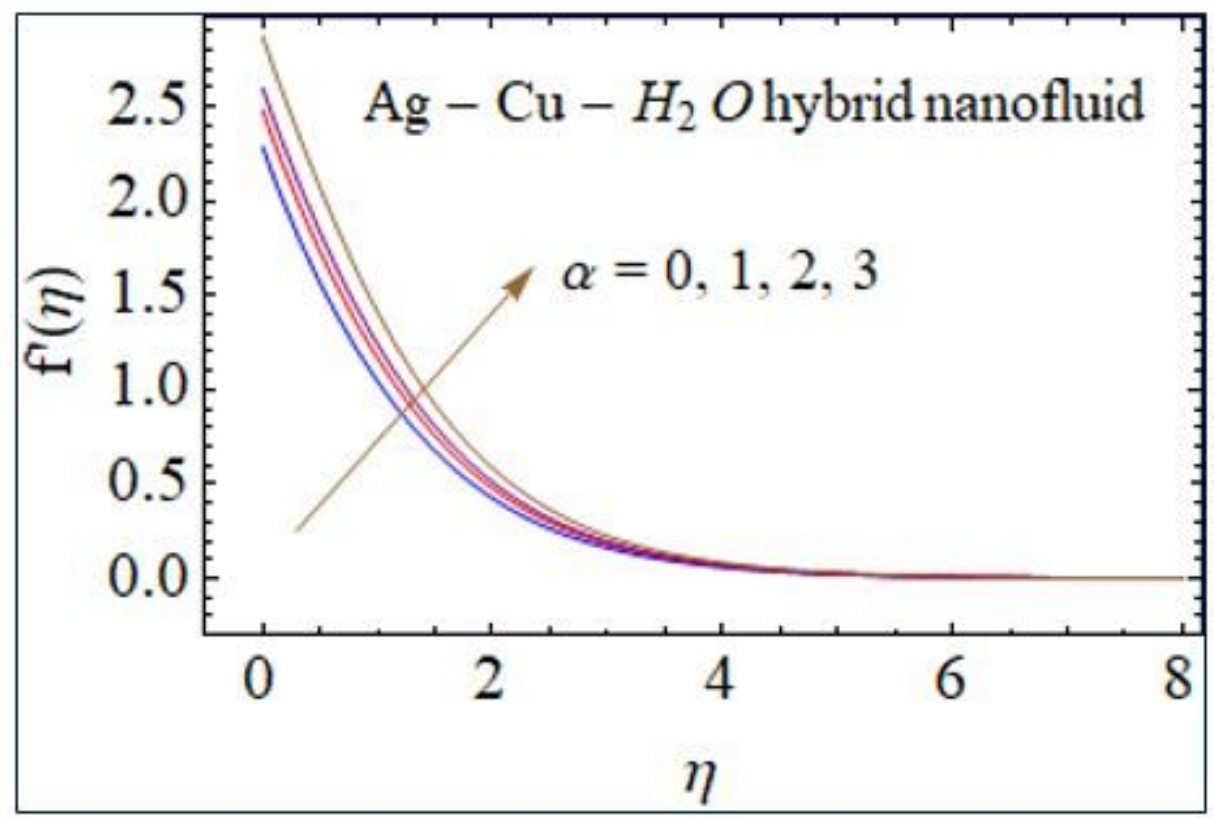

Figure 3

please see the manuscript file for the full caption

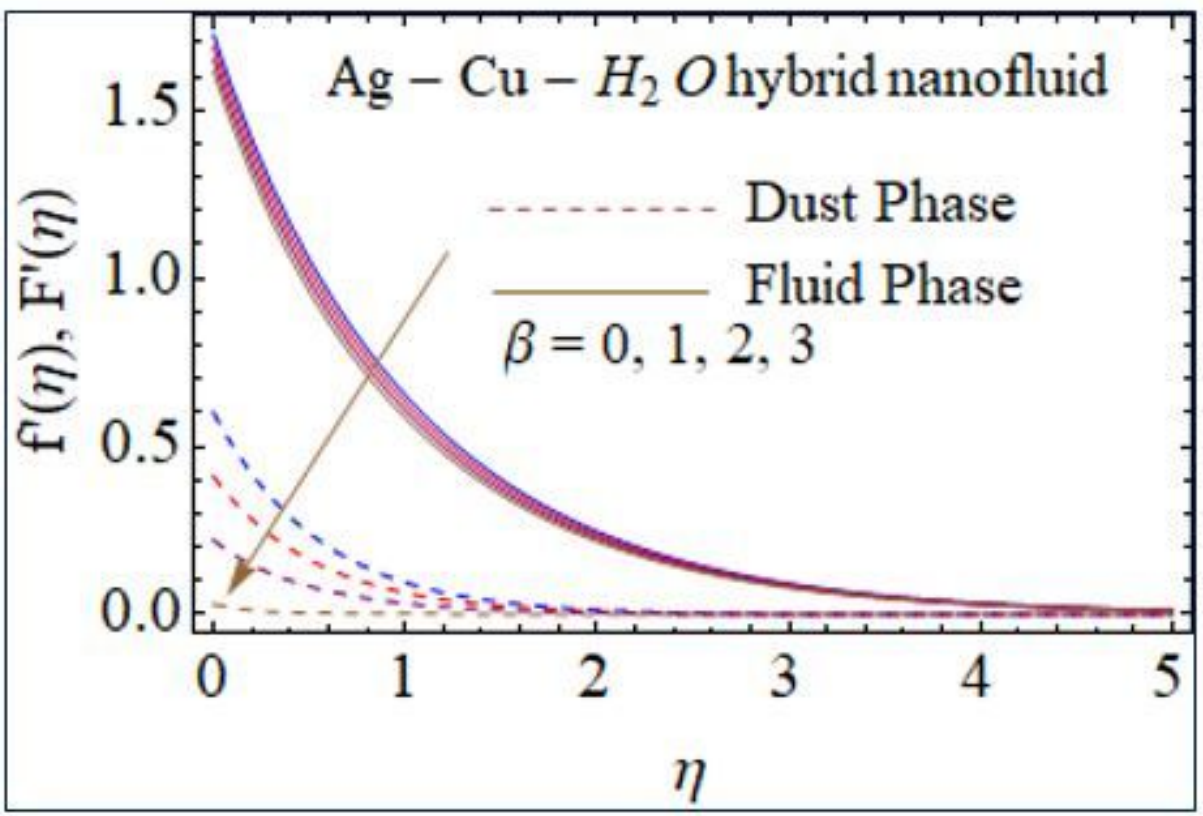

Figure 4

please see the manuscript file for the full caption 


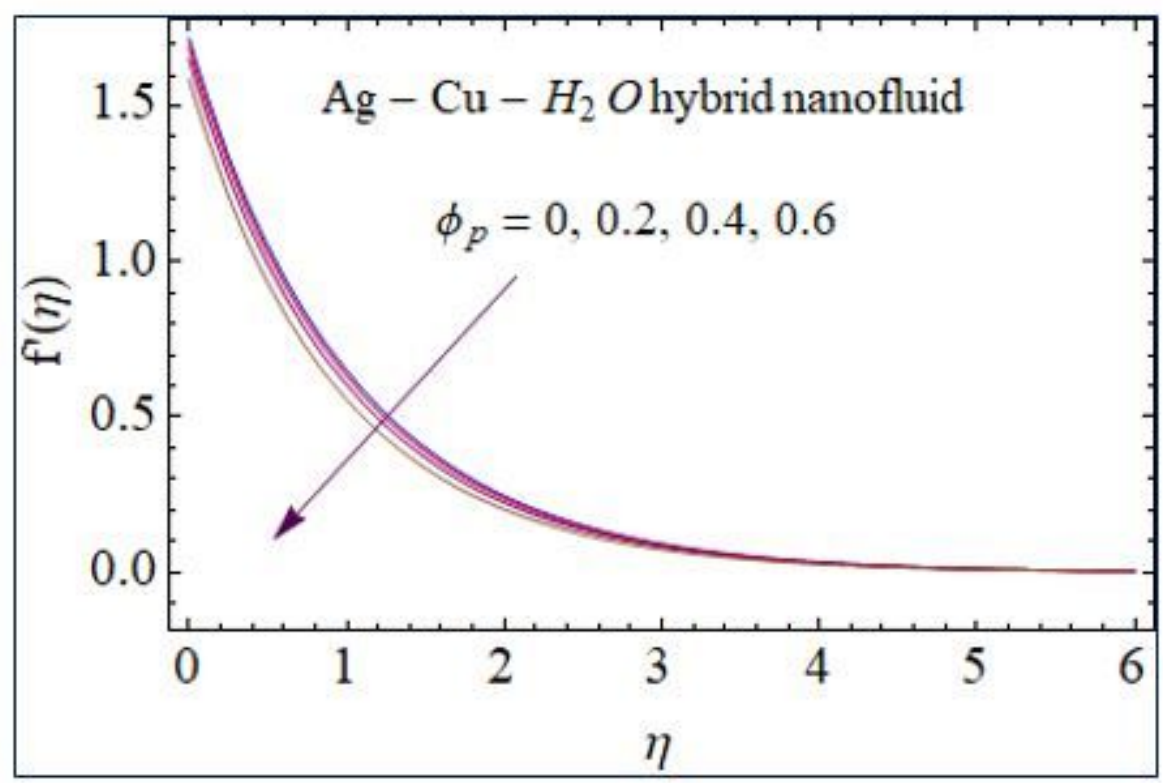

Figure 5

please see the manuscript file for the full caption

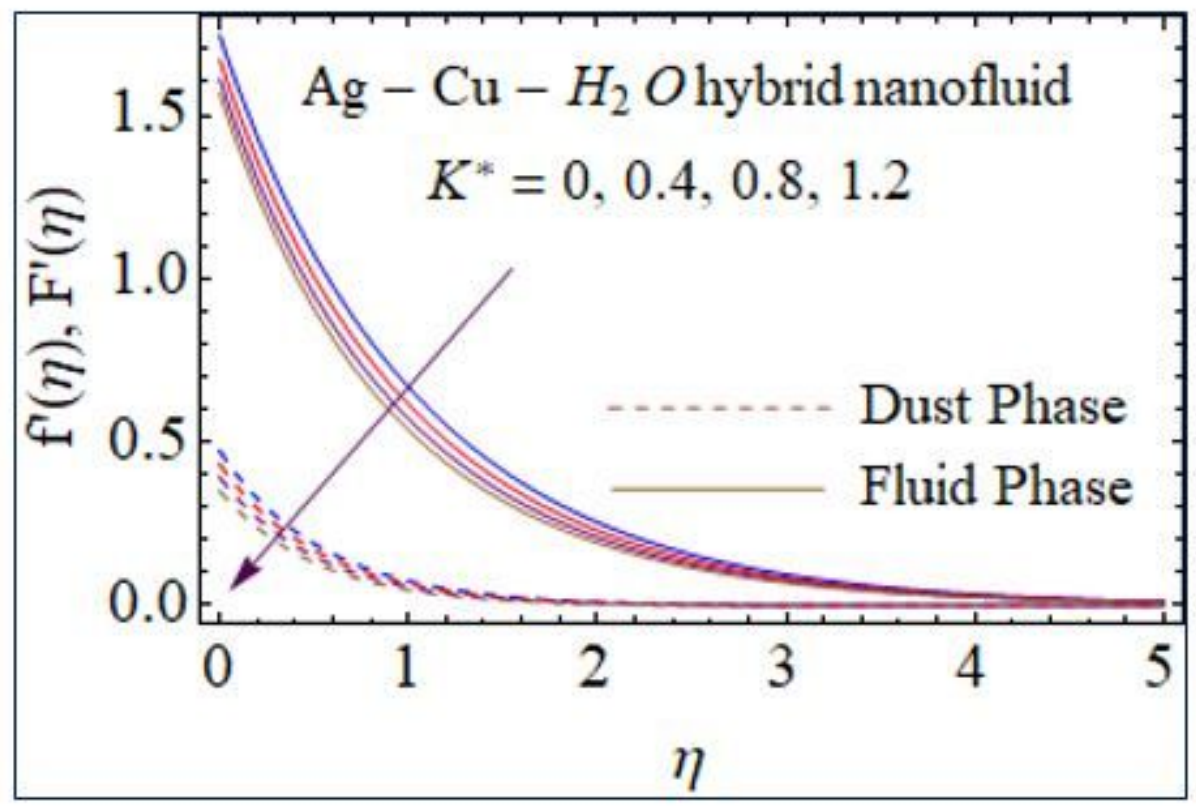

Figure 6

please see the manuscript file for the full caption 


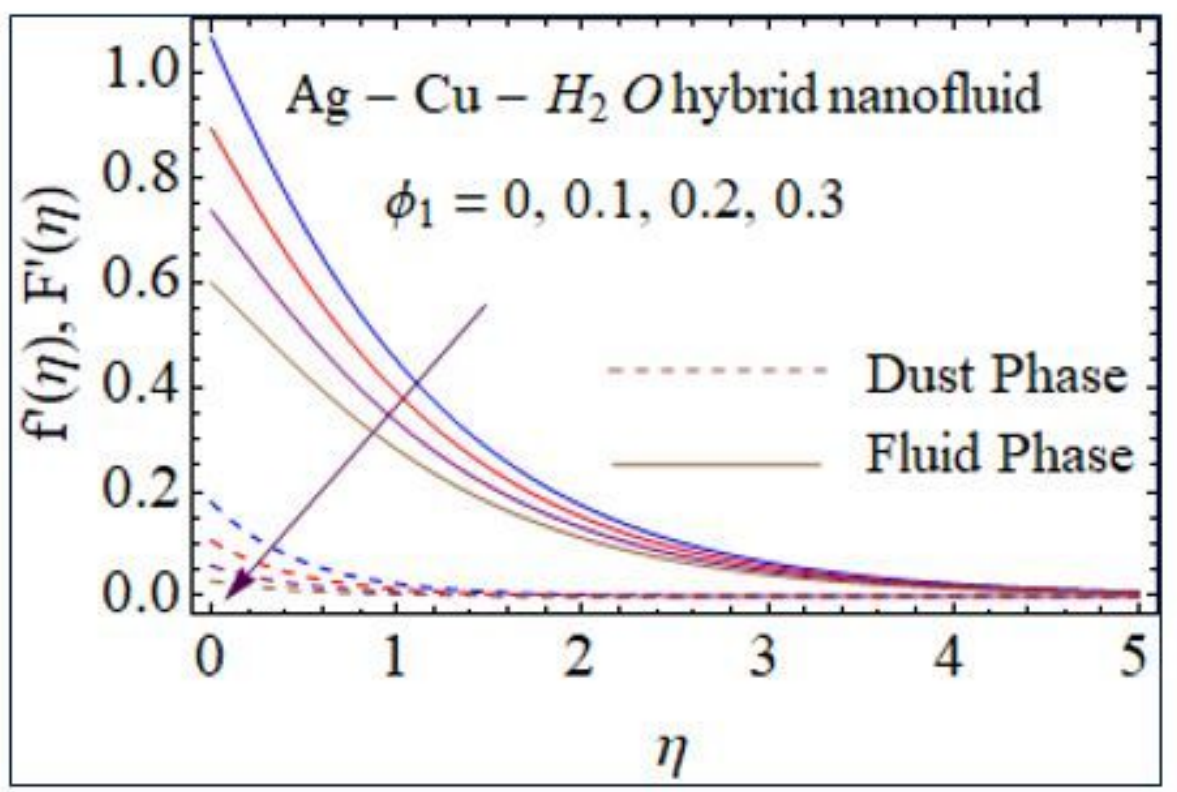

Figure 7

please see the manuscript file for the full caption

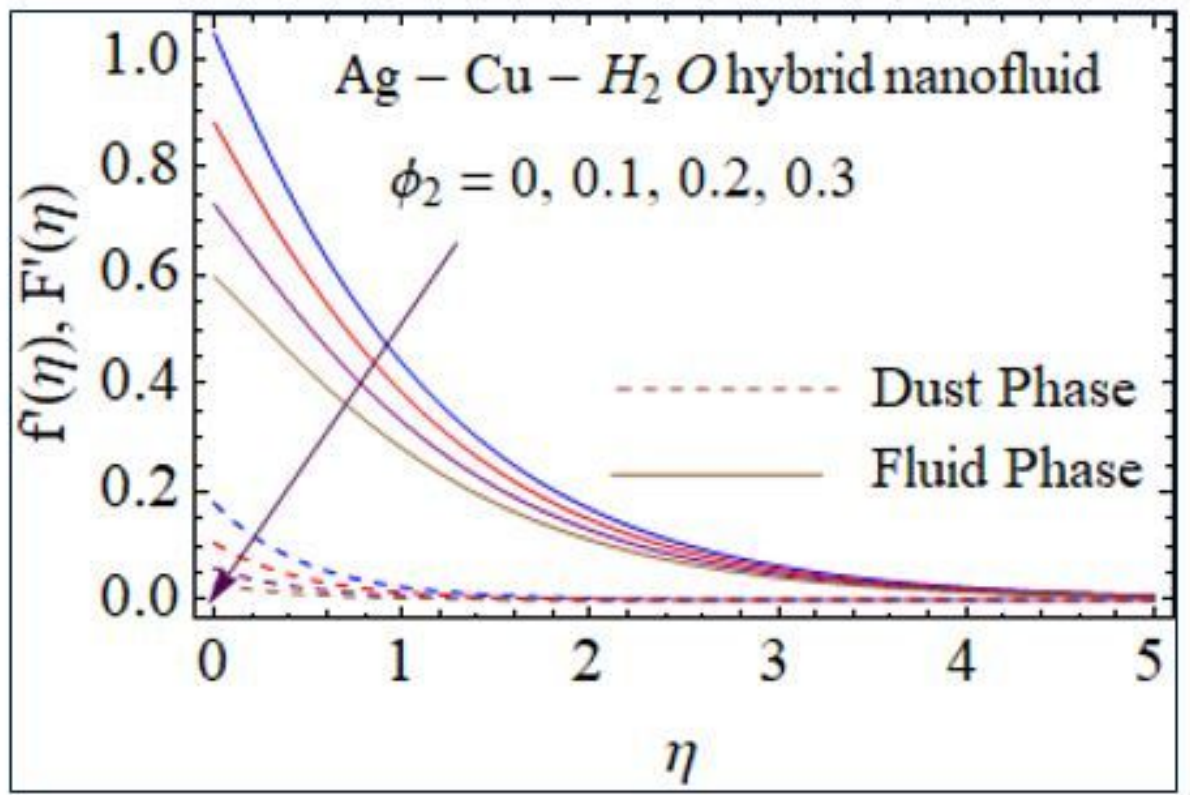

Figure 8

please see the manuscript file for the full caption 


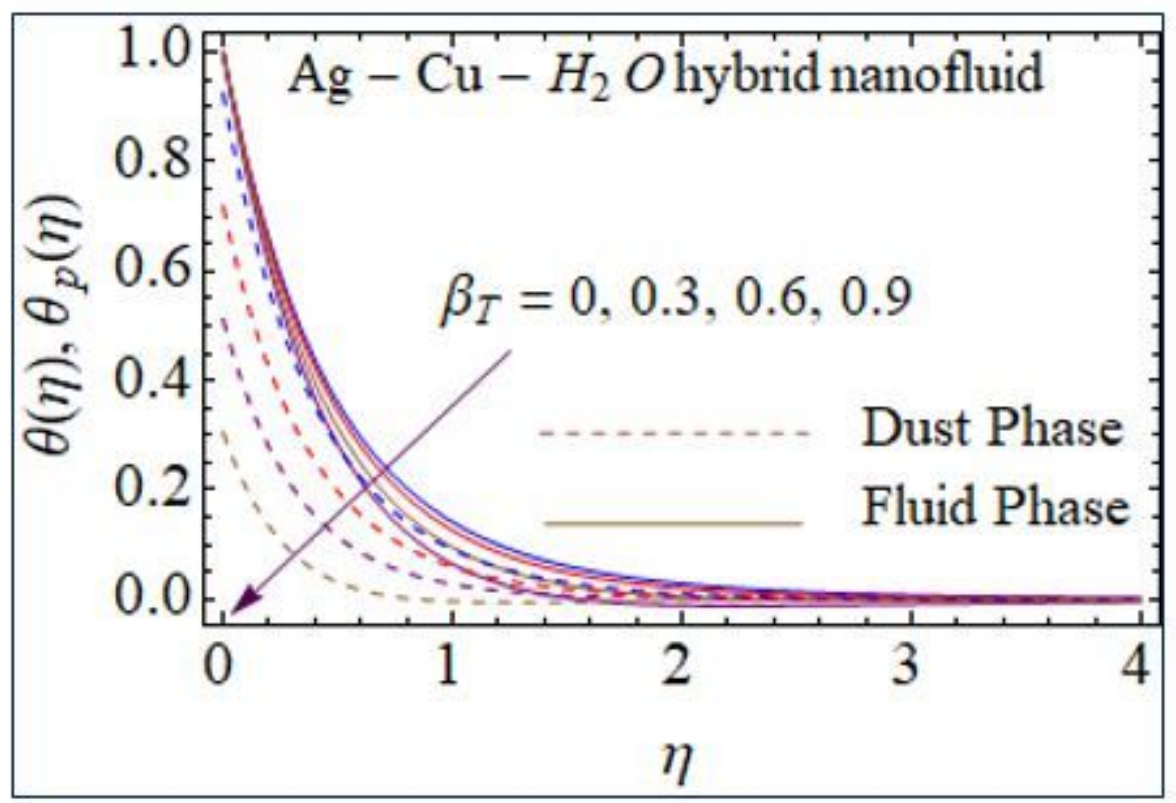

Figure 9

please see the manuscript file for the full caption

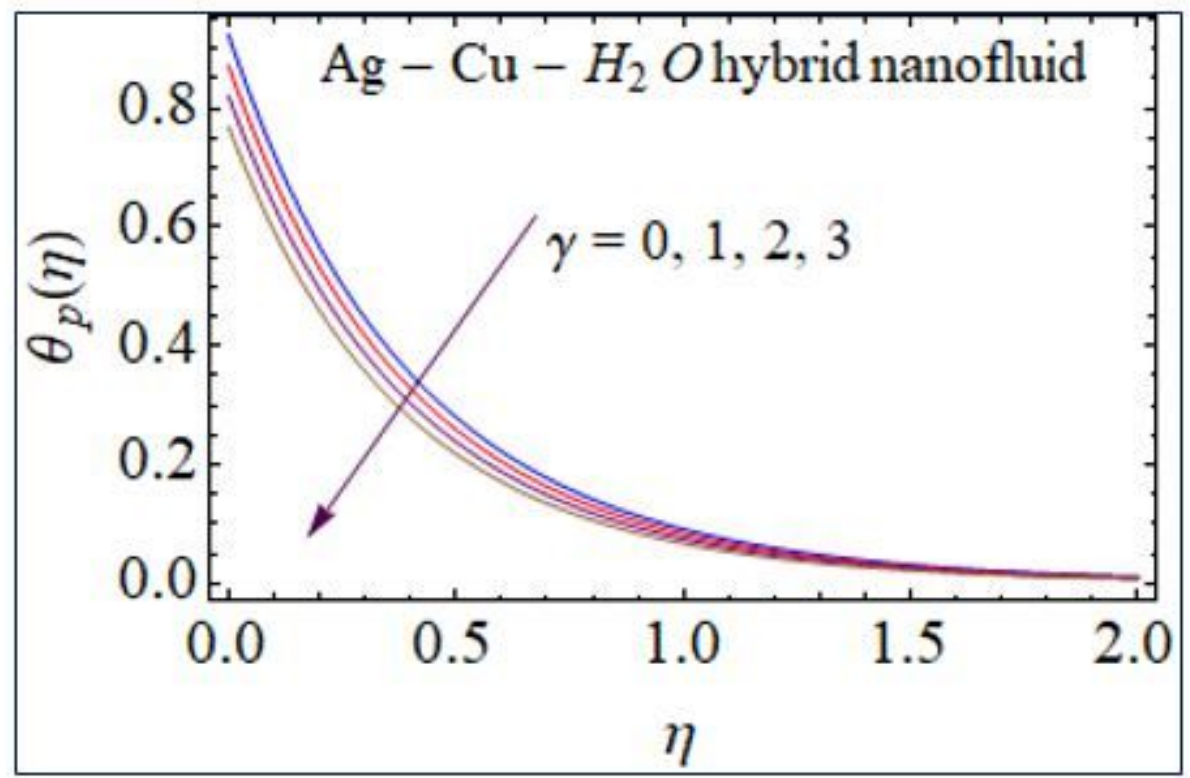

Figure 10

please see the manuscript file for the full caption 


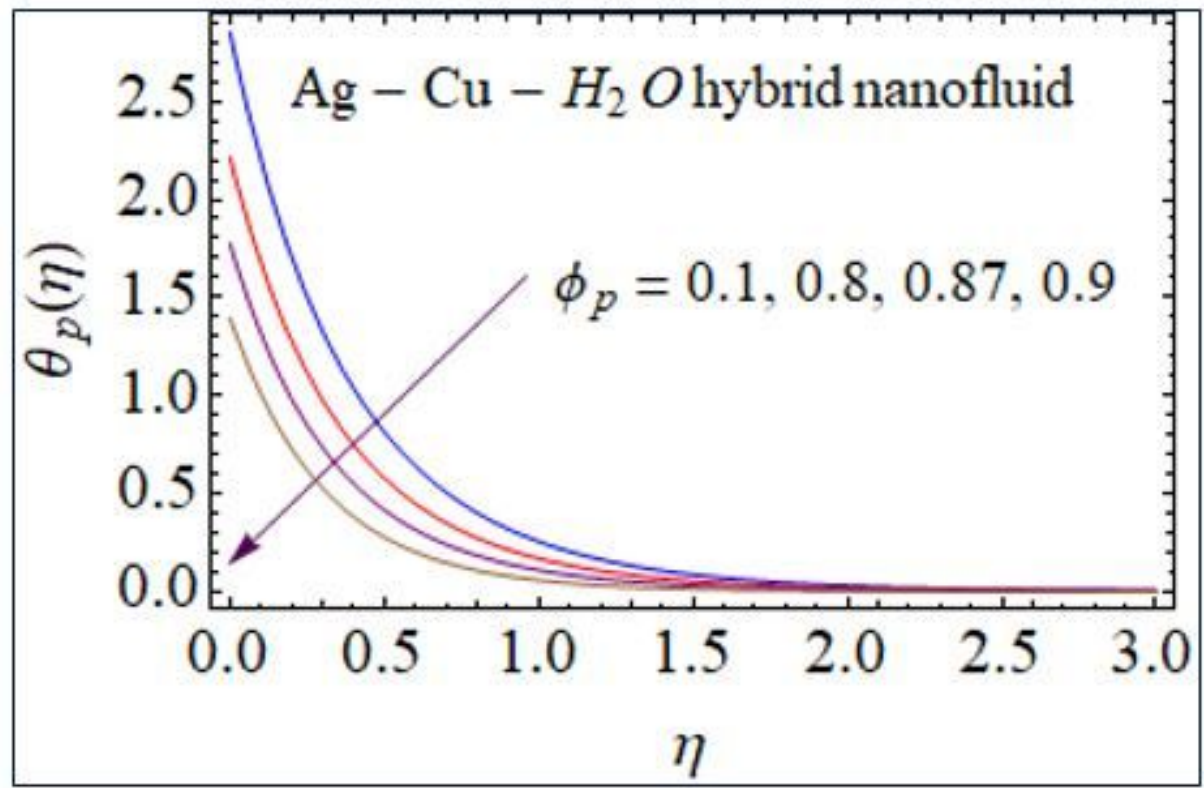

Figure 11

please see the manuscript file for the full caption

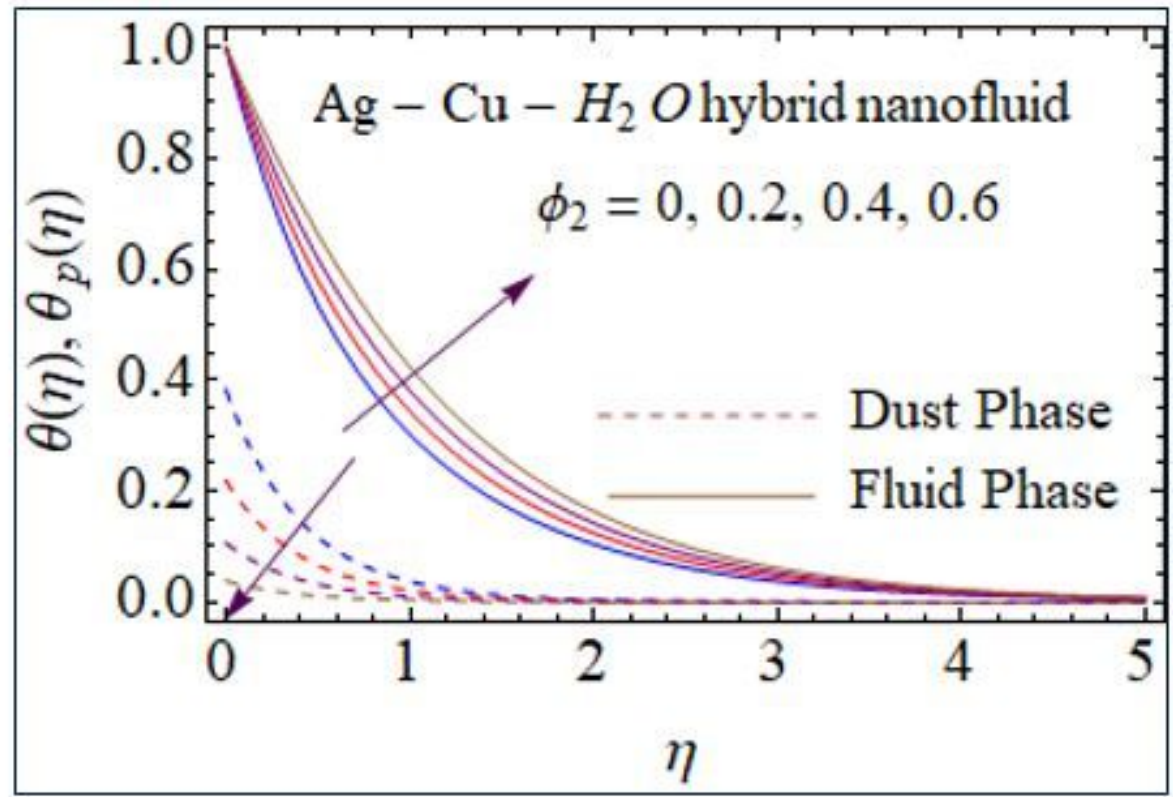

Figure 12

please see the manuscript file for the full caption 


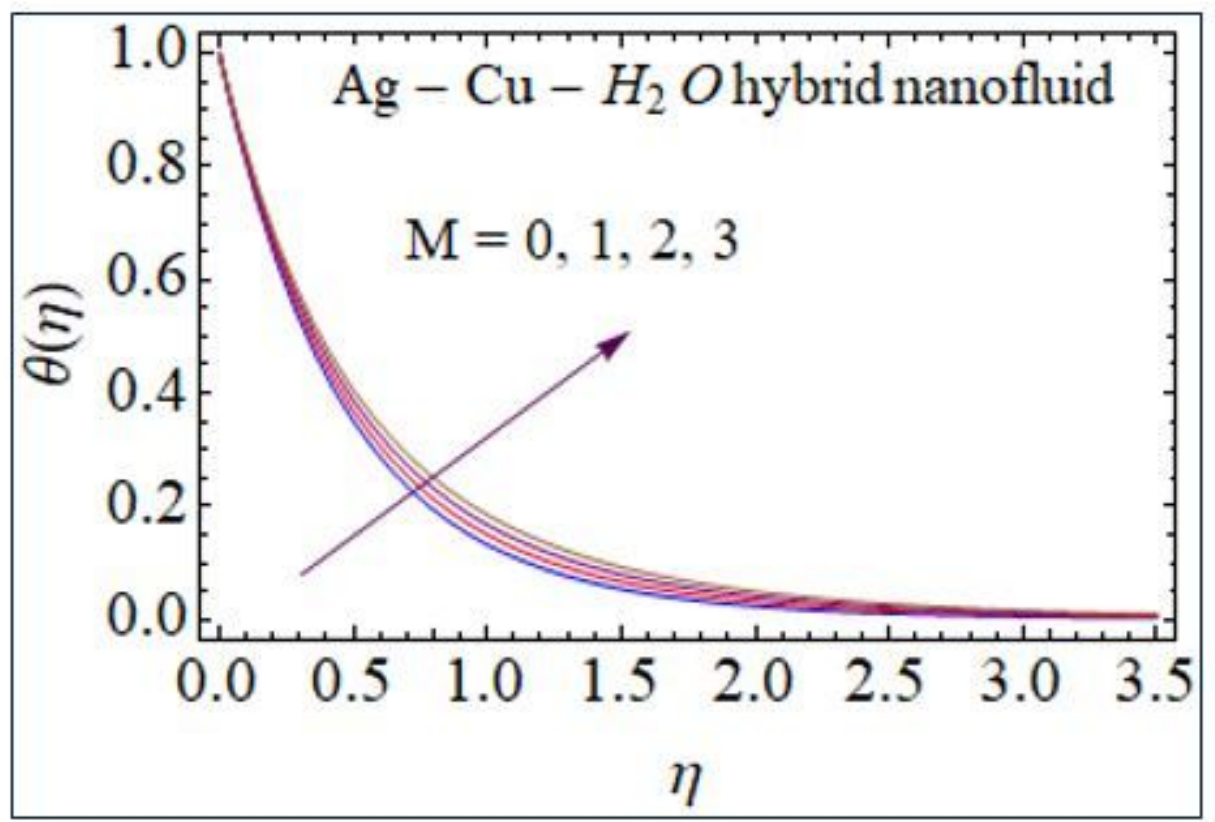

Figure 13

please see the manuscript file for the full caption 